hep-th/0605056

\title{
Finite level geometry of fractional branes
}

\author{
Albrecht Wurtz ${ }^{1}$ \\ Karlstads Universitet \\ Universitetsgatan 5 \\ S-651 88 Karlstad
}

\begin{abstract}
In some CFT models of simple current type, which are used to describe string theory on orbifolds and (adjoint) cosets of Lie groups, there arise fixed points of the simple current group. In these cases, the standard procedure to associate functions to Ishibashi states by averaging out the action of the simple current group, gives functions with unsatisfactory properties. In some cases the averaged Ishibashi function simply vanishes, which we see explicitly in $S O(3)$ at level $k=4 l+2$. In this note, an alternative function assignment is suggested, and it is shown that in some cases the resulting Ishibashi functions are orthogonal.
\end{abstract}

\footnotetext{
${ }^{1}$ albrecht . wurtz@kau.se
} 


\section{Introduction}

D-branes in string theory can be described in (at least) two different ways. One can, in the Lagrangian description, impose boundary conditions that restrict the end points of the string to live on certain subsets of the target space. Then, under certain conditions, the field theory on the world sheet is conformal [1, 2]. Alternatively, one can introduce boundary states in CFT and directly impose conformal invariance and other symmetry conditions. These boundary conditions can then (less directly) be given a geometrical interpretation in terms of functions on the target space. This function is interpreted as describing the localization of the string endpoint. In free boson theories, the boundary states turn out to correspond in a natural way to delta functions on the target space, in perfect agreement with the imposed boundary conditions in the Lagrangian description [3]. In WZW models, there is also an agreement between the descriptions, in the sense that the boundary states converge to certain delta functions in the limit of large level $[4]^{2}$. One would like to generalize this procedure to sigma models on orbifolds of Lie groups, and to coset models.

We consider boundary states that are rational, which are often symmetry preserving (they do not necessarily preserve the maximally extended symmetry). These boundary states can be used to construct a CFT for which correlators exist on all world sheets [6]. In particular, they satisfy the Cardy constraints. The Ishibashi blocks, commonly referred to as Ishibashi states, are obtained as a natural basis of solutions of the Ward identities. In the WZW theory we start from (which we will refer to as the covering theory), the target space is a connected, simply connected compact Lie group $G$. The Peter-Weyl theorem provides an isomorphism between the functions on $G$ and the horizontal descendants of the primary fields. This isomorphism associates group characters to Ishibashi blocks [4, which gives the boundary state a shape that converges, in the limit of large level, to a delta distribution on the group [4. In CFT, the Ishibashi blocks are solutions to the Ward identities, thus it is natural that they are associated to group characters (that are invariant under $A d_{G}$ ). Further, the characters are orthogonal with respect to the Haar measure on $G$, which reflects the corresponding orthogonality of the Ishibashi blocks.

We consider the shape of boundary states in a target space $Q$ which is a $\Gamma$-orbifold of $G$ (we shall refer to $G$ as the covering of $Q$ ), where $\Gamma$ is a finite group. The Ishibashi blocks in the corresponding CFT are constructed with simple current methods [7] 6]. We would like to associate functions to these. A priori, it is not clear that this is at all possible. After all, in string theory, D-branes are more than just the sub-manifolds of target space where the string end-point is located. Here, our focus is only on those aspects of the D-branes which concern their position in target space. From this point of view, it would be preferable (if possible) to associate functions to Ishibashi blocks.

One procedure to associate functions to Ishibashi blocks is to average out the action of $\Gamma$ on the Ishibashi functions on $G$, cf. [8, 9]. (Or, equivalently, one averages the functions associated to the boundary states over the action of the orbifold group.) This gives functions that are manifestly invariant under the orbifold group action, and therefore descend to functions on

\footnotetext{
${ }^{2}$ However, in the Lagrangian description, there are so-called exceptional branes with lower dimensionalities than the generic ones cf. 1], unlike in the CFT description [4, 5].
} 
the orbifold space $G / \Gamma$. However, when there are fixed points, this procedure gives Ishibashi functions of which some are vanishing, or associates the same function to different Ishibashi blocks. This mismatch is unsatisfactory, because the Ishibashi blocks should be orthogonal [10], and it is natural to expect the same for the associated functions.

The origin of this mismatch is the fact that in the CFT description, the Ishibashi blocks are not labeled with the same labels as the primary fields (to which functions are naturally associated). Instead we have pairs $\left(\Lambda_{f}, J\right)$, satisfying certain conditions, cf. [11, 7]. Here, $\Lambda_{f}$ is a label of a primary field in the covering theory, $J$ is a simple current used to define the orbifold, and $J * \Lambda_{f}=\Lambda_{f}$. The symbol $*$ denotes the fusion product, under which the set of simple currents is a group.

For concreteness, we consider the orbifold $S U(2) / \mathbb{Z}_{2}=S O(3)$. There is a simple current $k$ and a fixed point $\frac{k}{2} ; k * \frac{k}{2}=\frac{k}{2}$. We face two problems :

I) At level $k=4 l+2$, the label $m=k / 2$ occurs as (part of) an allowed Ishibashi label, namely $(k / 2, k)$, but the function associated to this Ishibashi block by the averaging procedure vanishes.

II) If instead $k=4 l$, we have two allowed Ishibashi labels $(k / 2, k)$ and $(k / 2,0)$, which are both associated to the same function with the averaging procedure.

In both cases we run into trouble with the orthogonality of the Ishibashi blocks [10]

$$
\left\langle\left\langle I_{m, J} \mid q^{L_{0}+\bar{L}_{0}-c / 12} I_{n, K}\right\rangle\right\rangle=\delta_{n m} \delta_{J K} \chi_{n}\left(q^{2}\right)
$$

functions which are linearly dependent cannot be orthogonal with any sensible scalar product. In the Lagrangian description, one associates the structure of a gerbe module to the branes [1]. Branes that are supported at the same sub-manifolds may differ by nothing more than their gerbe module structure, and this happens precisely when there are fixed points in the CFT description.

The main purpose of the present paper is to find a description that resolves the ambiguity II at finite values of the level. In section 22, a procedure to associate functions to Ishibashi blocks is suggested for rank $r=1$, (2.3). For higher rank groups, a universal procedure (2.5) is suggested and its implications are analyzed. The ansatz (2.5) is natural because it makes makes use of the isomorphism (see [15] eq. (14.110)) between the center $Z \subset G$ and the relevant simple current group $\mathcal{G}$. Further, it provides a universal procedure to associate functions to Ishibashi blocks such that the functions are (in many cases, including infinite series) orthogonal, as required. In the final part, we also propose a way to resolve the ambiguity I. In section 3 . the implications of (2.3) are investigated. In the fourth and final section, the diagonal coset model $S U(2)_{k} \times S U(2)_{l} / S U(2)_{k+l}$ is discussed.

\section{Ishibashi functions}

Choose a maximal torus $H \subset G$, and let $h \in \overline{\mathfrak{h}}^{*}=\operatorname{Lie}(H)^{*}$. For $x \in \mathbb{C}$, a representation function at $g \mathrm{e}^{\mathrm{i} x h}$ is related to its value at $g$ as

$$
D_{a b}^{\Lambda}\left(g \mathrm{e}^{\mathrm{i} x h}\right)=\left\langle\Lambda ; a\left|R^{\Lambda}\left(g \mathrm{e}^{\mathrm{i} x h}\right)\right| \Lambda ; b\right\rangle=\mathrm{e}^{\mathrm{i} x(b, h)} D_{a b}^{\Lambda}(g),
$$


where $a, b$ label states in the representation $\Lambda$ and $(\cdot, \cdot)$ is the Killing form (where we take the weight part of the label $b$, in case there are multiplicities). Recall [12] that the functions $D_{a b}^{\Lambda}$ are orthogonal in all three indices with the usual scalar product (integration with the Haar measure). A character at this argument can be written as

$$
\chi_{\Lambda}\left(g \mathrm{e}^{\mathrm{i} x h}\right)=\sum_{a \prec \Lambda} \mathrm{e}^{\mathrm{i} x(a, h)} D_{a a}^{\Lambda}(g) .
$$

The sum is over all states $a$ (with multiplicities) in the representation $\Lambda$.

Let us, to begin with, focus on the case $S O(3)=S U(2) / \mathbb{Z}_{2}$. The two elements of the simple current group $\mathbb{Z}_{2}$ are denoted 0 and $k$, and their action under fusion is $0 * j=j$ and $k * j=k-j$. The simple current group has a fixed point under the simple current action; $k * k / 2=k / 2$. When $k=4 l$, this fixed point corresponds to a primary field in $S O(3)$. Then we have Ishibashis which are 2 -fold degenerate and are labeled by pairs $(\Lambda, J)$ with the degeneracy $J \in\{0, k\}=\left\{k^{n}\right\}$ with $n \in\{0,1\}$ and $\Lambda=k / 2$ is the fixed point. Denote by $|\Lambda|$ the dimension of the (horizontal) $\mathfrak{s u}(2)$-representation with highest weight $\Lambda$. One can associate to the Ishibashi labeled $(\Lambda, J)$ the function

$$
\left.\left\langle g \mid I_{\Lambda, J}\right\rangle\right\rangle:=\sqrt{\frac{1}{|G|}} \chi_{\Lambda}\left(g \mathrm{e}^{\mathrm{i} \pi \sigma_{3} \frac{n}{\Lambda \Lambda}}\right) .
$$

Here, $G=S U(2)$ and $|G|=2 \pi^{2}\left(k \alpha^{\prime}\right)^{3 / 2}$ denotes the volume of the group [13]. For both values of $n,(2.3)$ defines a function on $S O(3)$. We shall see that they are not only linearly independent, but in fact orthogonal. Consider the scalar product of two Ishibashis with different labels $n=0$ and $n^{\prime}=1$, calculated via their functions,

$$
\begin{aligned}
\int_{G} \operatorname{dg}\left\langle\left\langle I_{\Lambda, 0} \mid g\right\rangle\left\langle g \mid I_{\Lambda, 1}\right\rangle\right\rangle & =\frac{1}{|G|} \int_{G} \operatorname{dg} \sum_{a, b \prec \Lambda} \mathrm{e}^{\mathrm{i} \pi a /|\Lambda|} D_{a a}^{\Lambda}(g) D_{b b}^{\Lambda}(g)^{*} \\
& =\frac{1}{|\Lambda|} \sum_{a \prec \Lambda} \mathrm{e}^{\mathrm{i} \pi a /|\Lambda|}=0 .
\end{aligned}
$$

Recall $|\Lambda|=\Lambda+1$ and $a=-\Lambda,-\Lambda+2, \ldots, \Lambda-2, \Lambda$, so the sum is a sum over roots of unity $\mathrm{e}^{\mathrm{i} \pi a /|\Lambda|}$. Ishibashis with different degeneracy labels are now orthogonal, as they should be, see [10] eq. (4.40). Moreover, the normalization of the individual Ishibashis is left unchanged. This is the prescription to resolve degenerate Ishibashi functions that will be applied in the coming section.

The rest of this section is on generalizing the above procedure to higher rank groups. The simple currents of the WZW models (with one exception, which occurs for $E_{8}$ ) are labeled by $k$ times the fundamental weights $\Lambda_{(i)}$ with $a_{i}=1$ [14]. These are the cominimal weights, cf. the table in [14], p 203. The $\Lambda_{(i)}$ are the highest weights of the fundamental representations, and $\Lambda_{(0)}$ is the unit element in the fusion ring, hence also of the simple current group. $E_{8}$ at level $k=2$ has an additional simple current $\Lambda_{(7)}$. In all cases except $E_{8}$ at level $k=2$, there exists an isomorphism between the simple current group and the center of the group; $\mathcal{G} \cong Z(G)$, given by $g_{(J)}^{\xi}=\exp \left(\xi \mathrm{i} H_{\Lambda_{(J)}}\right)$ with $\xi=2 \pi$, cf. [15]. Here the $H_{\Lambda_{(J)}}$ are appropriately normalized so that the exponential of a fundamental Weyl alcove is a fundamental domain of the $A d_{G}$-action on $G$. When $\Lambda=\Lambda_{f}$ is a fixed point $\Lambda_{(i)} * \Lambda_{f}=\Lambda_{f}$, it makes sense to require 
$I_{\left(\Lambda_{f}, \Lambda_{(0)}\right)}(g)=\chi_{\Lambda_{f}}(g)$. We also require that we have an action of the simple currents on the Ishibashis, $I_{\left(\Lambda_{f}, \Lambda_{(J)} * \Lambda_{(0)}\right)}(g)=I_{\left(\Lambda_{f}, \Lambda_{(0)}\right)}\left(g g_{(J)}^{\xi}\right)$, as in the case without fixed points [ [5]. In the light of the simple current - center isomorphism, the most general ansatz would appear to be $I_{\left(\Lambda_{f}, \Lambda_{(L)}\right)}(g)=\chi_{\Lambda_{f}}\left(g g_{(L)}^{2 \pi}\right)$. However, as $g_{(L)}^{2 \pi} \in Z(G), \chi_{\Lambda}\left(g g_{(L)}^{2 \pi}\right)$ is a class function, which we know is a linear combination of characters. We require that the Ishibashi functions are linearly independent. For given $\Lambda$, there are $d_{\Lambda}^{2}$ orthogonal representation functions (by the theorem of Peter and Weyl, see [12]). Guided by the isomorphism $\mathcal{G} \cong Z(G)$ as discussed above, we associate functions

$$
I_{\left(\Lambda_{f}, \Lambda_{(i)}\right)}(g):=\sqrt{\frac{1}{|G|}} \chi_{\Lambda_{f}}\left(g \mathrm{e}^{\mathrm{i} H_{\Lambda_{(i)}} \xi}\right), \quad \xi \neq 2 \pi .
$$

to the Ishibashi blocks. We shall now investigate whether the prescription (2.5) leads to Ishibashi functions which are also orthogonal (with the right choice of $\xi$ ), in which case the basis for Ishibashis suggested in [10] is indeed orthogonal. The scalar product between two Ishibashis with $\Lambda_{f}$ a fixed point, is

$$
\begin{aligned}
\left\langle I_{\left(\Lambda_{f}, \Lambda_{(i)}\right)}(g), I_{\left(\Lambda_{f}, \Lambda_{(j)}\right)}(g)\right\rangle & =\sum_{a \prec \Lambda, b \prec \Lambda^{\prime}} \mathrm{e}^{\mathrm{i}\left(a, \Lambda_{(i)}\right) \xi} \mathrm{e}^{-\mathrm{i}\left(b, \Lambda_{(j)}\right) \xi} \int_{G} d g D_{a a}^{\Lambda_{f}}(g) D_{b b}^{\Lambda_{f}^{\prime}}(g)^{*} \\
& =\frac{1}{\left|\Lambda_{f}\right|} \bar{\chi}_{\Lambda_{f}}\left(\Lambda_{(i)} \xi-\Lambda_{(j)} \xi\right) .
\end{aligned}
$$

Thus, the questions whether these Ishibashis are orthogonal reduces to a calculation of the corresponding horizontal Lie algebra character $\bar{\chi}_{\Lambda_{f}}\left(\Lambda_{(i)} \xi-\Lambda_{(j)} \xi\right)$.

When we consider the fixed point $\Lambda=m \rho$ (which appears in all Lie groups at level $k=m g^{\vee}$ ), it is useful to rewrite the Lie algebra character with the Weyl character formula

$$
\left\langle I_{\left(\Lambda, \Lambda_{(i)}\right)}(g), I_{\left(\Lambda, \Lambda_{(j)}\right)}(g)\right\rangle=\frac{1}{|\Lambda|} \frac{\sum_{\sigma \in W}|\sigma| \exp \left(\mathrm{i} \xi \sigma(\Lambda+\rho), \Lambda_{(i)}-\Lambda_{(j)}\right)}{\sum_{\sigma \in W}|\sigma| \exp \left(\mathrm{i} \xi \sigma(\rho), \Lambda_{(i)}-\Lambda_{(j)}\right)}
$$

If $\Lambda_{(i)}=\Lambda_{(j)}$, we have a character evaluated at the origin, which takes the value $|\Lambda|$. Otherwise, one can show that $\Lambda_{(i)}-\Lambda_{(j)}=\omega\left(\Lambda_{(k)}\right)$ for some fundamental weight $\Lambda_{(k)} \neq 0$, and some Weyl group element $\omega \in W$. To prove this, we need to do a case by case analysis where [16] is a useful reference. For illustration, consider $E_{6}$, which has a $\mathbb{Z}_{3}$ simple current group with cominimal weights $\Lambda_{(1)}$ and $\Lambda_{(5)}$. In terms of fundamental Weyl reflections $w_{i}(\lambda)=\lambda-\lambda^{i} \alpha_{(i)}$ one finds (e. g. by an algorithm suggested in [17]) that

$$
\Lambda_{(1)}-\Lambda_{(5)}=w_{5} w_{4} w_{3} w_{2} w_{6} w_{3} w_{4} w_{5}\left(\Lambda_{(5)}\right) .
$$

Similarly, one can show in all other cases as well that for all simple currents $\Lambda_{(i)}, \Lambda_{(j)}$ the difference is a horizontal Weyl element acting on a fundamental weight $\Lambda_{(k)} \neq 0$.

We use this fact to proceed with our calculation of the Ishibashi function scalar product,

$$
\left\langle I_{\left(\Lambda, \Lambda_{(i)}\right)}(g), I_{\left(\Lambda, \Lambda_{(j)}\right)}(g)\right\rangle=\frac{1}{|\Lambda|} \frac{\sum_{\sigma \in W}|\sigma| \exp \left[\mathrm{i} \xi(m+1)\left(\rho, \sigma\left(\Lambda_{(k)}\right)\right)\right]}{\sum_{\sigma \in W}|\sigma| \exp \left[\mathrm{i} \xi\left(\rho, \sigma\left(\Lambda_{(k)}\right)\right)\right]}
$$


We can now use the denominator identity to rewrite both the denominator and the numerator,

$$
\left\langle I_{\left(\Lambda, \Lambda_{(i)}\right)}(g), I_{\left(\Lambda, \Lambda_{(j)}\right)}(g)\right\rangle=\frac{1}{|\Lambda|} \frac{\prod_{\alpha>0} \sin \left(\frac{1}{2} \xi\left(\alpha,(m+1) \Lambda_{(k)}\right)\right)}{\prod_{\alpha>0} \sin \left(\frac{1}{2} \xi\left(\alpha, \Lambda_{(k)}\right)\right)}
$$

The product is over the positive roots. Some factors in both products vanish because $\left(\alpha^{j}, \Lambda_{(k)}\right)=$ 0 for simple $\alpha^{j}$ with $j \neq k$. This happens at both sides of the fraction, thus we shall ignore those factors. The other factors where $\alpha$ contains $\alpha^{k}$ are the ones which do not vanish for all $\xi$. We shall choose $\xi$ such that

$$
\sin \left(\frac{1}{2}(m+1) \xi\left(\alpha^{k}, \Lambda_{(k)}\right)\right)=0 \quad \text { and } \quad \sin \left(\frac{1}{2} \xi\left(\alpha^{k}, \Lambda_{(k)}\right)\right) \neq 0 .
$$

Then the Ishibashis have the desired orthogonality. We set

$$
\xi \equiv \frac{2 \pi}{(m+1)\left(\alpha^{k}, \Lambda_{(k)}\right)} .
$$

All $\left(\alpha^{k}, \Lambda_{(k)}\right)$ are equal for those $\Lambda_{(k)}$ that appear in $\Lambda_{(i)}-\Lambda_{(j)}=\omega\left(\Lambda_{(k)}\right)$, hence the Ishibashis are indeed orthogonal. Thus, for these Lie groups, our prescription yields a natural basis of Ishibashi functions that are orthogonal in the labels $m \rho, \Lambda_{(i)}$. These Ishibashi functions can then be used to build the boundary state functions, which describe the shape of the D-branes. This will be displayed in detail for $S U(2) / \mathbb{Z}_{2}=S O(3)$ below.

As an example of a fixed point that is not of the form $\Lambda=m \rho$, we consider $S U(4)$ at level $k=4$ where the simple current $\Lambda_{(2)}$ has the fixed point $\Lambda_{f}=2 \Lambda_{(0)}+2 \Lambda_{(2)}$, the horizontal part of which is $\bar{\Lambda}=(0,2,0)$. The character is

$$
\bar{\chi}_{(0,2,0)}\left(\mathrm{i} \xi \Lambda_{(2)}\right)=10+2 \cos (2 \xi)+8 \cos (\xi),
$$

which does not vanish for any (real) value of $\xi$. As the simple current group has only two elements, and precisely one of the functions (2.5) associated to the fixed point Ishibashi labels is a class function, it is clear that the two functions are linearly independent. But to obtain an orthogonal basis of Ishibashis, it appears that we must take a linear combination of $\left\{\Lambda_{(0)}, \Lambda_{(2)}\right\}$ to label the degeneracy. The simple current $\Lambda_{(2)}$ also has the fixed point $\Lambda_{f}=2 \Lambda_{(1)}+2 \Lambda_{(3)}$; for this fixed point, the corresponding horizontal character does vanish with some choice of $\xi$. Thus, $\left\{\Lambda_{(0)}, \Lambda_{(2)}\right\}$ provides in this case an orthogonal basis of degeneracy labels.

That the Ishibashis can be made orthogonal for $\Lambda_{f}=(2,0,2)$, but not for $\Lambda_{f}=(0,2,0)$, is related to the fact that the former representation has more states (84 instead of 20). Since the states in the representations are distributed on regular polygons, a large representation will typically have a smaller percentage of states orthogonal to the axis $\Lambda_{(2)}$. Thus, we expect the failure of the Ishibashi functions to be orthogonal in the $(0,2,0)$ case to be a small level phenomenon, which is supported by an analysis of other cases. For example, in $S U(6)$ at level $k=2$, the current $\Lambda_{(3)}$ has 3 fixed points of dimensions 20, 84 and 84 . An analysis of the orthogonality of the Ishibashi functions (2.5) gives similar conclusions as in the case $S U(4)$ at level $k=4$. 
The missing function As already mentioned, in $S O(3)=S U(2) / \mathbb{Z}_{2}$ at level $k=4 l+2$, we get a label $\kappa$ for the Ishibashi block which cannot in an obvious way be related to a unique function on the quotient space: Since $k / 2$ is odd, the Ishibashi label $k / 2$ does not naturally correspond to a representation of the Lie group $S O(3)$. However, we can take a fundamental domain (of the $\Gamma$-action) $D \subset M$ in the covering space $M$ (which in our applications is a Lie group, but the considerations are general). On $M$, we do have a natural Ishibashi function $I_{\kappa}^{M}$ for that label. Now we can take the restriction $\left.I_{\kappa}^{M}\right|_{D}$, and extend it to a function $\tilde{I}_{\kappa}$ on $M$ that is invariant under the orbifold group $\Gamma$ (and which is different from the function $I_{\kappa}^{M}$ ). This function projects to a candidate for the Ishibashi function $I_{\kappa}^{Q}$ on the quotient $Q=M / \Gamma$. Since the integration $\left\langle I_{\kappa}, I_{\Lambda}\right\rangle_{Q}$ can be lifted to $D$, and if $\left\langle I_{\kappa}, I_{\Lambda}\right\rangle_{D}=0$ for $\kappa \neq \Lambda$, the Ishibashi functions are orthogonal. We shall spell out explicitly how this works for $M=S U(2)$ and $\Gamma=\mathbb{Z}_{2}$ in the next section, and we shall see that the suggested function is indeed orthogonal to the other Ishibashi functions on $S O(3)$.

\section{$3 \quad$ Ishibashis of $\mathrm{SO}(3)$}

The CFT $S O(3)_{k}$ can be described as a simple current extension of $S U(2)_{k}$ when $k=4 l$, and as a permutation invariant when $k=4 l+2$. The primary fields in the covering $S U(2)_{k}$ are labeled $j=0,1, \ldots, k$. The simple current group is $\mathcal{G}=\mathbb{Z}_{2}=\{k, 0\}$. The nontrivial element acts via fusion as $k * j=k-j$ and its fixed point is $k / 2$. The Ishibashis are labeled $(m, J)$ with

$$
m=J * m, \quad Q_{\mathcal{G}}(m)+X(\mathcal{G}, J) \in \mathbb{Z},
$$

cf. [7. Since $\mathcal{G}$ is cyclic, the discrete torsion is trivial and $X$ vanishes except for $X(k, k)=$ $\frac{1}{2} Q_{k}(k)=\frac{k}{4} \bmod \mathbb{Z}$. When $k=4 l+2$, we have discrete torsion in the sense $X \neq 0$ (but not in the sense of a freedom of choice in $X$ ). There are two types of Ishibashi labels in the simple current construction; the regular type

$$
(m, 0) \quad \text { with } \quad m \in 2 \mathbb{Z},
$$

and one Ishibashi of exceptional type

$$
\left(\frac{k}{2}, k\right) \quad \text { with } \quad k \in 2 \mathbb{Z} .
$$

Both species are always present, and the first type Ishibashis are labeled by the allowed primary fields. The Ishibashis are expected to be orthogonal to each other, [10, and therefore the function cannot only depend on more the first entry in the pair $(m, J)$. Thus, the associated function cannot simply be the character $\chi_{m}(g)$ averaged over the orbifold group; instead (2.3) is suggested. Alternatively, we take (2.5) with $\xi$ given by (2.12), which reduces to (2.3) in this case. Recall that the group manifold $S O(3)=S U(2) / \mathbb{Z}_{2}$ is the set of equivalence classes

$$
[g]=[z g] \quad g \in S U(2) \quad z=-\mathbf{1}=\mathrm{e}^{\mathrm{i} \pi \sigma_{3}} .
$$

The stabilizer of this identification is trivial for all $g$. A function on $S O(3)$ is a function on the covering for which $f(z g)=f(g)$. In the case $k=4 l+2$ we have

$$
D_{m m}^{k / 2}(z g)=-D_{m m}^{k / 2}(g) .
$$


Simply averaging a sum of these over the orbifold group gives an everywhere vanishing function. Therefore, the group character $\chi_{k / 2}(g)$ cannot be projected to $S O(3)$ by this averaging procedure. Instead, we may proceed as follows: for each $[g] \in S O(3)$ pick a representative $g$ that lies in the upper hemisphere of $S U(2)$, which is the set of points with $\psi<\pi / 2$ in the parametrization

$$
g=\cos \psi \mathbf{1}+\sin \psi \bar{\sigma} \cdot \bar{n}
$$

The character $\chi_{k / 2}$ (which is an odd function of $\psi$ on $S U(2)$ ), can be modified to an even function $\tilde{\chi}_{k / 2}$ on $S U(2)$ by defining $\tilde{\chi}_{k / 2}:=\chi_{k / 2}$ on the upper hemisphere, and $\tilde{\chi}_{k / 2}:=-\chi_{k / 2}$ on the lower hemisphere. Note that $\chi_{k / 2}=0$ on the equatorial plane. To the Ishibashi labelled $(k / 2, k)=(2 l+1, k)$, we associate

$$
I_{(2 l+1, k)}([g])=\sqrt{\frac{1}{|G|}} \tilde{\chi}_{k / 2}(g)
$$

This function is orthogonal to all $S O(3)$-characters: the allowed $S O(3)$-characters are of the form $\chi_{2 n}$ and are even functions as well, thus we may evaluate the scalar product by just integrating over the upper hemisphere. Then we can use invariance of the measure to obtain

$$
\left\langle\tilde{\chi}_{k / 2}, \chi_{2 l}\right\rangle=\int_{0}^{\pi / 2} d \mu_{\psi} \chi_{k / 2}(\psi) \chi_{2 l}(\psi)-\int_{\pi / 2}^{\pi} d \mu_{\psi} \chi_{k / 2}(\psi) \chi_{2 l}(\psi)=0 .
$$

A similar calculation reveals that $\left\langle\tilde{\chi}_{k / 2}, \tilde{\chi}_{k / 2}\right\rangle=\left\langle\chi_{k / 2}, \chi_{k / 2}\right\rangle$.

Boundary states Recall that the boundary labels are orbits $\left[j, \psi_{j}\right]$, where $j$ is an $S U(2)$ label that representats a $\mathcal{G} \cong \mathbb{Z}_{2}$ orbit, and $\psi_{j}$ is a character of $C_{j} \subset S_{j}$. The subgroup $C_{j}$ of the stabilizer $S_{j}$ is in our case given by $C_{j}=S_{j}$. Further, $C_{k / 2}=\mathbb{Z}_{2}$, and all other $C_{j}$ are trivial. Hence $\psi_{j}$ is a degeneracy label that takes values \pm 1 . A list of boundary labels is (supressing trivial labels)

$$
\left[j, \psi_{j}\right]=[0],[1], \ldots,[k / 2-1],[k / 2,1],[k / 2,-1]
$$

The boundarry states $[j]$ with odd $j$ do not correspond to primary fields of $S O(3)_{2 k}$ and are interpreted as being symmetry-breaking. There are $k / 2+2$ states in this list, just as many as there are Ishibashis (3.2) and (3.3). The boundary sates are linear combinations of the characters with coefficients [7]

$$
B_{(m, J),\left[j, \psi_{j}\right]}=\sqrt{\frac{|\mathcal{G}|}{\left|S_{j}\right|\left|C_{j}\right|}} \frac{\alpha_{J} S_{m, j}^{J}}{\sqrt{S_{0, m}}} \psi_{j}(J)^{*}=\frac{\sqrt{2}}{\left|S_{j}\right|} \frac{\alpha_{J} S_{m, j}^{J}}{\sqrt{S_{0, m}}} \psi_{j}(J)^{*},
$$

which are known as the boundary coefficients. The matrix $S^{J}$ is different from the modular matrix $S$ only if $m=j=k / 2$. In that case, $S_{k / 2, k / 2}^{k}=\frac{1}{k} \mathrm{e}^{-3 \pi \mathrm{i} k / 8}$. The phase $\alpha_{J}$ can be taken to be $\alpha_{k}=\mathrm{e}^{\mathrm{i} \pi / 4}$ when $k=4 l+2$ and unity in all other cases. 
Shape of the boundary states Now we are ready to compute some of these boundary shapes. At level $k=4 l$, we have a fractional boundary state with $j=k / 2=2 l$. With (2.3), the shape of the fractional boundary state is

$$
\begin{aligned}
B_{[2 l, \psi]}(g) & =\frac{1}{\sqrt{2}} \sum_{(m, J)} \frac{S_{m, 2 l}^{J}}{\sqrt{S_{0, m}}} \psi(J) I_{(m, J)}(g) \\
& =\frac{1}{\sqrt{2}} \sum_{m=0,2, \ldots, k} \frac{S_{m, 2 l}}{\sqrt{S_{0, m}}} \chi_{m}(g)+\psi(k) \frac{1}{4 l \sqrt{2}} \frac{\mathrm{e}^{-3 \pi \mathrm{i} l / 2}}{\sqrt{S_{0,2 l}}} \chi_{2 l}\left(\mathrm{e}^{\frac{\mathrm{i} \pi}{2 l+1} \sigma_{3}} g\right),
\end{aligned}
$$

where $\psi(k)= \pm 1$. The shift $g \mapsto \mathrm{e}^{\frac{\mathrm{i} \pi}{2 l+1} \sigma_{3}} g$ is interpreted as a tilting of the conjugacy class by an angle $\pi /(2 l+1)$. In [9] and [8] the $\psi$-dependent last term does not contribute with a full group character.

\section{The coset $[\mathrm{SU}(2) \times \mathrm{SU}(2)] / \mathrm{Ad}(\mathrm{SU}(2))$}

The prescription (2.3) can be applied to coset models with nontrivial field identification fixed points, in particular to the coset

$$
Q=\frac{S U(2) \times S U(2)}{\operatorname{Ad}(S U(2))}
$$

which is the set of equivalence classes $\left[g_{1}, g_{2}\right]=\left[g g_{1} g^{-1}, g g_{2} g^{-1}\right]$ with $g, g_{1}, g_{2} \in G$. We use the parametrization

$$
Q=\left\{(t, q) \mid t \in T_{W}, \quad \begin{array}{l}
q \in T_{W} \text { if } t= \pm e \\
q \in G / \operatorname{Ad}(T) \text { else }
\end{array}\right\} .
$$

The CFT description of a sigma model with target space an adjoint coset $Q=G / \operatorname{Ad}(H)$, where $H \subset G$, goes as follows. The primary fields are labelled by certain pairs $(j, m)$ where $j$ is a primary in $G$ and $m$ a primary in $\bar{H}$. Here $\bar{H}$ is a certain CFT related to $H$ (the modular tensor category describing the primary fields of $\bar{H}$ is the dual of the MTC associated to $H$ [18]). Not all pairs $(j, m)$ of labels appear in the coset, there are selection rules and field identifications, which (for non-Maverick cosets) can be obtained by a simple current construction. The relevant simple current group (in this setting called identification group) is the one that geometrically corresponds to $Z_{G} \cap H$. In our model (4.1), the identification group is generated by $(-e,-e) \in$ $G \times G$, which corresponds to $\mathbb{Z}_{2}=\{(0,0,0),(k, l, k+l)\}$. The monodromy of a label with respect to this current is required to be integer, which leads to the selection rule

$$
a+b-c \in 2 \mathbb{Z} \text {. }
$$

The identification group has a fixed point if both levels $k$ and $l$ are even, which then is $(k / 2, l / 2, k / 2+l / 2)$.

Now we wish to describe the shape of the boundary states in this model. Most of the analysis is similar as in the case without fixed points (cf. [5]), only that we need something like (2.3) to associate functions to the degenerate Ishibashis. One can find the Ishibashi and 
boundary labels by following the standard procedure. The self- monodromy of the non-trivial identification current is two times its conformal weight, which is always integer. Hence the discrete torsion $X$ vanishes. Therefore, the Ishibashis are labeled by tuples $(m, J)$ where the monodromy of $m$ with respect to $J$ vanishes, and $J * m=m$. Thus, the first species of Ishibashis are simply the primary fields allowed by (4.3). For even levels $k, l \in 2 \mathbb{Z}$ there will be an additional fractional Ishibashi labeled

$$
(m ; K)=(k / 2, l / 2, k / 2+l / 2 ; k, l, k+l) .
$$

In order to associate functions to primary fields, it is convenient to first rewrite the (adjoint) coset (4.1) as a set of equivalence classes [5, 19]

$$
[g, h]_{l r}=[u g v, u h v]_{l r}, \quad u, v \in H .
$$

To the Ishibashis labeled $(a, b, c ; 0)$ we associate a function which is invariant under the coset action;

$$
I_{(a, b, c, 0)}\left[u g_{1} v, u g_{2} v, u h v\right]=I_{(a, b, c, 0)}\left[g_{1}, g_{2}, h\right],
$$

which is achieved by the function [5], eq. $(5.7)^{3}$. Applied to the present context, the formula in [5] involves the Clebsch-Gordan coefficients of $S U(2)$. In our notation $c$ is contained in $a \times b$ if $c=|a-b|, \ldots, a+b$, and the magnetic quantum number $\gamma$ is in the representation $c$ if $|\gamma| \leq c$, with multiplicity given by the Clebsch-Gordan coefficient $c_{\gamma, \alpha, \beta}^{c \prec a \times b}$. Recall that the Clebsch-Gordan coefficients are defined by

$$
c_{m, m_{1}, m_{2}}^{j \prec j_{1} \times j_{2}}=\left\langle j, m\left|\left(\left|j_{1}, m_{1}\right\rangle \otimes\left|j_{2}, m_{2}\right\rangle\right) .\right.\right.
$$

Note that $c_{\gamma, \alpha, \beta}^{c \prec a \times b}=0$ if $\gamma \neq \alpha+\beta$. Also note that $\sum_{\alpha, \beta, \gamma}\left|c_{\gamma, \alpha, \beta}^{c \prec a \times b}\right|^{2}=d_{a} d_{b}=d_{c}$. The regular Ishibashi function after integrating out the coset action is

$$
I_{a, b, c}^{Q}\left[g_{1}, g_{2}, h\right]=\sqrt{\frac{\left|G_{k+l}\right|^{5}}{\left|G_{k}\right|\left|G_{l}\right|}} \sqrt{\frac{d_{a} d_{b}}{d_{c}^{3}}} \sum_{\substack{\delta, \epsilon \prec a \\ \mu, \nu \prec b}} D_{\delta \epsilon}^{a}\left(g_{1}\right) D_{\mu \nu}^{b}\left(g_{2}\right) D_{\delta+\mu, \epsilon+\nu}^{c}(h)^{*}\left(c_{\delta+\mu, \delta, \mu}^{c \prec a \otimes b}\right)^{*} c_{\epsilon+\nu, \epsilon, \nu}^{c \prec a \otimes b} .
$$

The shape of the fractional Ishibashi is the analoguous projection of the product of twisted characters,

$$
\begin{aligned}
I_{K}^{Q}\left[g_{1}, g_{2}, h\right] & =\sqrt{\frac{\left|G_{k+l}\right|^{5}}{\left|G_{k}\right|\left|G_{l}\right|}} \sqrt{\frac{d_{a} d_{b}}{d_{c}^{3}}} \\
& \times \sum_{\substack{\delta, \epsilon \prec a \\
\mu, \nu \prec b}} D_{\delta \epsilon}^{k / 2}\left(g_{1} \mathrm{e}^{\mathrm{i} \sigma_{3} 2 \pi / k}\right) D_{\mu \nu}^{l / 2}\left(g_{2} \mathrm{e}^{\mathrm{i} \sigma_{3} 2 \pi / l}\right) D_{\delta+\mu, \nu+\epsilon}^{(k+l) / 2}\left(h \mathrm{e}^{\mathrm{i} \sigma_{3} 2 \pi /(k+l)}\right)^{*}\left(c_{\delta+\mu, \delta, \mu}^{c \prec a \otimes b}\right)^{*} c_{\epsilon+\nu, \epsilon, \nu}^{c \prec a \otimes b} .
\end{aligned}
$$

The Ishibashi function (4.9) is orthogonal to the other Ishibashi functions (4.8), because the scalar product can be lifted to the covering $G \times G \times G$ where orthogonality follows from previous considerations. The shapes of the boundary states can now be calculated by using the boundary coefficients given in [7. The results of this calculation are more complicated than enlightening; we refrain from presenting them here.

\footnotetext{
${ }^{3}$ There is a small error in that formula: the Ishibashi function should be without the averaging over the simple current group. This distinction is insignificant in the context of [5], where the focus is on boundary state functions; the sum over the identification group comes in in the next step, so the boundary functions obtained in [5] are still correct.
} 
Acknowledgements The author would like to thank Jürgen Fuchs for helpful comments.

\section{References}

[1] K. Gawedzki, Abelian and non-Abelian branes in the WZW models and gerbes, Commun. Math. Phys. 258 (2005) 23-73, http://xxx.lanl.gov/abs/hep-th/0406072

[2] U. Schreiber, C. Schweigert and K. Waldorf, Unoriented WZW Models and Holonomy of Bundle Gerbes, ZMP-HH/05-28, Hamburger Beitr. zur Mathematik Nr. 228 http://xxx.lanl.gov/abs/hep-th/0512283

[3] P. Di Vecchia, M. Frau, I. Pesando, S. Sciuto, A. Lerda, R. Russo, Classical p-branes from boundary state, Nucl. Phys. B 507 (1997) 259-276 http://xxx.lanl.gov/abs/hep-th/9707068

[4] G. Felder, J. Fröhlich, J. Fuchs and C. Schweigert The geometry of WZW branes, J. Geom. Phys. 34 (2000) 162-190 http://xxx.lanl.gov/abs/hep-th/9909030

[5] J. Fuchs and A. Wurtz, On the geometry of coset branes, Nucl.Phys. B724 (2005) 503-528, http://xxx.lanl.gov/abs/hep-th/0505117

[6] J. Fjelstad, J. Fuchs, I. Runkel, C. Schweigert, TFT construction of RCFT correlators V: Proof of modular invariance and factorisation, to appear in Theory and Applications of Categories, http://xxx.lanl.gov/abs/hep-th/0503194

[7] J. Fuchs, L. Huiszoon, A. Schellekens, C. Schweigert and J. Walcher, Boundary coefficients and simple currents and Ishibashi labels, Phys.Lett. B495 (2000) 427-434 http://xxx.lanl.gov/abs/hep-th/0007174

[8] J. Maldacena, G. Moore and N. Seiberg, Geometrical interpretation of D-branes in gauged WZW models, JHEP 0107 (2001) 046, http://xxx.lanl.gov/abs/hep-th/0105038

[9] P. Bordalo and A. Wurtz, D-branes in lens spaces, Phys. Lett. B 568 (2003) 270-280 http://xxx.lanl.gov/abs/hep-th/0303231

[10] J. Fuchs, C. Schweigert, Symmetry breaking boundaries I, General theory, Nucl.Phys. B558 (1999) 419-483, http://xxx.lanl.gov/abs/hep-th/9902132

[11] L. Huiszoon, D-branes and O-planes in string theory, PhD thesis, Amsterdam 2002.

[12] T. Bröcker, T. tom Dieck, Representaions of compact Lie groups, Springer 1985

[13] C. Bachas, M. Douglas, C. Schweigert Flux stabilization of D-branes, JHEP 0005 (2000) 339, http://xxx.lanl.gov/abs/hep-th/0003037

[14] J. Fuchs, Affine Lie algebras and quantum groups, Cambridge University Press, 1992

[15] P. Di Franscesco, P. Mathieu, D. Senechal, Conformal Field Theory, Springer 1997 
[16] R. King, A. Al-Qubanchi, The Weyl groups and weight multiplicities of the exceptional Lie groups, J. Phys. A: Math. Gen. 14 (1981) 51-75

[17] R. Capps, The Weyl orbits of $G_{2}, F_{4}, E_{6}$ and $E_{7}$, J. Phys. A: Math. Gen. 22 (1989) 1223-1243

[18] J. Fröhlich, J. Fuchs, I. Runkel, C. Schweigert, Correspondences of ribbon categories, Adv. Math. 199 (2006) 192-329, http://xxx.lanl.gov/abs/math.CT/0309465

[19] S. Fredenhagen and V. Schomerus, D-branes in coset models, JHEP 0202 (2002) 005, http://xxx.lanl.gov/abs/hep-th/0111189 